\title{
Menschen durchs Leben bis zum Tod begleiten
}

\author{
Das SÄZ-Podium zur ärztlichen Suizidhilfe vom 27. November in Basel ist auf grosses \\ Interesse gestossen, auch über die Berufsgruppe der Ärztinnen und Ärzte hinaus. \\ Hier der im Nachgang zur Podiumsdiskussion entstandene Beitrag eines Theologen \\ und ehemaligen Pfarrers.
}

\section{Paul Kohler}

Pensionierter Pfarrer der ref. Kirchgemeinde Pratteln-Augst

Korrespondenz:

Paul Kohler

Dürrenmattweg 4

CH-4133 Pratteln

kohler.pratteln[at]bluewin.ch

\section{Nicht gehaltener Diskussionsbeitrag}

Als einer, der nicht zur Zunft gehört, hätte ich mich nur zu Wort gemeldet, wenn mehr Zeit zur Verfügung gestanden hätte. Eine Woche später habe ich aufgeschrieben, was ich eigentlich hätte sagen müssen:

Mit der auf dem Podium gemachten und wohl allgemein anerkannten Aussage «Der Hausarzt, bzw. die Hausärztin begleitet den Patienten durchs Leben bis zum Tod» bin ich sehr einverstanden. So verstehe ich meine Aufgabe als Pfarrer auch, wobei diese Begleitung wie bei den Spezialärzten oft nur während eines kurzen Lebensabschnittes stattfindet (aber deswegen nicht weniger angebracht und wertvoll ist).

Ich bin aber nicht einverstanden, wenn diese ärztliche Begleitung den Freitod ausschliesst, wie es Frau Professor Stoppe gesagt hat. Denn es ist keine Sünde, seinem Leben durch einen bewussten Entschluss ein Ende zu setzen, schon gar nicht, wenn dieser Entschluss aus einem unerträglichen und nicht therapierbaren körperlichen und/oder seelischen Leiden entstanden ist.

Als Pfarrer will ich die, die sich mir anvertrauen, bedingungslos begleiten, sei es bei einer Ehe-Scheidung oder bei einer Todes-Scheidung. Beides sind keine leicht gefassten Entschlüsse. Wir machen alle bei solchen schweren Entscheidungen einen Reifeprozess durch, sowohl die Ehepartner bzw. die Freitodwilligen und deren Angehörige. Und nicht zuletzt wir, die sie beraten und begleitet haben.

Deshalb ist es wichtig, dass Sie mit der ärztlichen Begleitung nicht vor dem Freitod aufhören. Die Sterbehilfe schliesst eine eventuelle Suizidhilfe mit ein. Darunter verstehe ich Gespräche mit der Patientin oder dem Patienten, die über längere Zeit andauern; ferner das Schreiben der aktuellen Diagnose/Prognose und die Bestätigung der Urteilsfähigkeit der sterbewilligen Person. Ob Sie bereit sind, auch das Rezept für das Sterbemittel Natrium-Pentobarbital auszustellen, müssen Sie nach Ihrem Gewissen entscheiden. Niemand kann Sie dazu verpflichten. Wenn Sie das Rezept nicht ausstellen, hat Ihr Patient sicher dafür Verständnis, zumal er ja die Möglichkeit hat, sich an einen Vertrauensarzt einer Sterbehilfeorganisation zu wenden. Entscheidend für eine ärztliche Begleitung im Blick auf einen begleiteten Freitod ist aber das partnerschaftlich geführte Gespräch.

\section{Theologische Begründung meiner positiven Einstellung zum Freitod}

Als reformierter Pfarrer habe ich keinen Einwand gegenüber dem begleiteten Suizid. Ich weiss, dass die Mehrheit der reformierten und katholischen Kollegen theologische Einwände vorbringen. Diese kann ich verstehen und will sie ernst nehmen, vor allem weil die Fragen über Leben und Tod ernste Fragen sind. Hingegen darf weder der Arzt noch der Pfarrer seine eigene Überzeugung als allgemeingültiges Dogma ausgeben und andere Menschen darauf verpflichten. Denn jeder Mensch ist sein eigener «Chairman» (Ruth Cohn) und berechtigt, über sein eigenes Leben selbst zu bestimmen. Allerdings muss auch gesagt werden, dass der grössere Teil der Menschheit lebenswichtige Entscheidungen (z.B. über Ausbildung, Berufswahl, Partnerwahl, Arztwahl, Religion, gnädiger Tod) nicht selbst treffen kann wegen der kulturellen, politischen und sozialen Verhältnisse, in die diese Menschen hineingeboren wurden und darin leben müssen. Das Thema «begleiteter Freitod» ist in erster Linie ein Problem des Nordens, wie auch alle Fragen, welche die künstliche Befruchtung und zu früh Geborenen betreffen.

Wie begründe ich als Theologe meine positive Einstellung zum begleiteten Freitod und somit auch zu den Sterbehilfeorganisationen?

1. Die Bibel, sowohl die jüdische Bibel (also das sog. Alte oder Erste Testament) als auch das Neue Testament berichtet insgesamt nur von 4 Suiziden: von König Saul und seinem Waffenträger; von Ahitophel, dem Ratgeber von König David, und von Judas, der den Aufenthaltsort von Jesus dem Hohenrat, der jüdischen Religionsbehörde in Jerusalem, verraten hat. Saul und sein Waffenträger stürzten sich ins Schwert, Ahitophel und Judas haben sich erhängt. Diese Suizide - es sind Verzweiflungssuizide - werden in der Bibel nicht kommentiert. Dass diese Suizide in der Bibel nicht verurteilt werden etwa im Unterschied zu vielen sexuellen Beziehungen und Praktiken, finde ich zwar sympathisch, doch es genügt nicht, meine Auffassung zu begründen.

2. Eine wichtige Hilfe sind mir die Postulate der Aufklärung, sich seines Verstandes zu bedienen und sich aus der Unmündigkeit zu befreien. Ich bin also bereit, ausnahmslos hinter alle Dogmen (nicht nur 
hinter die kirchlichen) ein Fragezeichen zu setzen und sie mit Hilfe der Vernunft kritisch zu hinterfragen. Es ist mir dabei klar, dass nicht alles mit der Logik erklärt werden kann. Zudem kann kein Mensch ohne Dogmen leben, seien diese nun religiös oder nicht-religiös.

3. Mein Glaube als Seelsorger gründet auf dem Namen IMMANU-EL. Dieser wird beim Propheten Jesaja genannt (Jes. 7, 14; 8, 8) und wird im Evangelium nach Matthäus auf Jesus angewendet (Mt 1, 23): «Man wird ihm den Namen IMMANU-EL geben». Die Übersetzung dieses hebräischen Wortes wird bei Matthäus gleich angefügt: «Das heisst «Gott mit uns`.»

Der Gott, an den ich glaube, der im Alten wie im Neuen Testament derselbe ist und in Jesus von Nazareth ein Mensch wie unsereiner geworden ist und deshalb die Höhen und Tiefen des menschlichen Daseins nicht nur kennt, sondern am Kreuz auch erleidet - dieser Gott (EL) ist mit uns (IMMANU). Er ist also kein Wegweiser, der stehen bleibt und uns allein ziehen lässt, sondern mit uns geht, auch wenn unser Weg mal nach rechts und mal nach links führt. Er geht mit auf Holzwegen, auf Abwegen, auch in den Abgrund und in den Tod, und sei dieser eine Folge von Krankheit, Unfall oder von Gewalt inkl. Suizid.

\section{Kein Arzt oder Pfarrer darf seine eigene Überzeugung als allgemeingültiges Dogma ausgeben.}

Weil ich an diesen mich in meinem Leben begleitenden Gott glaube, begründe ich darin auch mein Seelsorge-Verständnis. D.h., wenn die Menschen von Gott begleitet sind in gesunden Tagen wie im Leiden und Sterben, dann will/muss auch ich sie begleiten, ihnen beistehen, ihnen Mut machen, sie segnen auf allen ihren Wegen.

Und schliesslich: Was ist denn so schlimm, wenn ein schwer leidender Mensch stirbt, vor allem wenn er sich das sehnlichst wünscht, weil er endlich erlöst sein will? Sei es, dass er davon überzeugt ist, im Sterben in die gnädige Hand Gottes zu fallen und in eine höhere Welt zu gelangen, sei es, dass er an kein Weiterleben glaubt und froh ist, wenn er ewig schlafen kann und nie mehr aufwachen muss .

Während die Begleitung des Arztes in der Regel mit dem Tod des Patienten endet, ausser es ist erwünscht, die Angehörigen noch eine gewisse Zeit zu beraten, treffe ich mich als Pfarrer vor der Abdankung des Verstorbenen mit den Angehörigen zu einem Gespräch. In städtischen Verhältnissen sehe ich oft nicht nur die Angehörigen zum ersten Mal, sondern muss feststellen, dass ich auch den Verstorbenen nicht gekannt habe. Ich bin angewiesen auf das, was mir die Angehörigen über den Verstorbenen, seine Einstellung zum Leben und Sterben berichten.

Da habe ich mich hin und wieder gefragt: Weshalb muss ein Mensch durch sein Leiden (und sei es
z.T. selbst verschuldet) so mürbe, klein und hässlich werden, dass er um Erlösung fleht? Trifft diese dann durch den Tod ein, atmen die Angehörigen auf und sagen: «Jetzt ist er endlich erlöst!» Diese Feststellung hilft ihnen, in der (schon während der Leidenszeit begonnenen) Trauer den Verlust des Menschen auch von seiner Warte aus anzuschauen.

Erlösung kann auch Gott und Jesus zum Subjekt haben, also den IMMANUEL, der sogar nicht nur bis zum Tod mitgeht, sondern auch ins ewige Leben hinüber führt und dort präsent ist. Das ist die Botschaft, die ich bei der Abdankung auszurichten habe.

Und als Seelsorger begleite ich nach der Abdankung die Angehörigen, sofern sie es nötig haben, eine gewisse Zeit weiter. Dies ist besonders wichtig bei Suiziden, vor allem bei den harten, den schrecklichen. Da kommen sofort bei den Angehörigen Schuldgefühle auf, je nach Abschiedsbrief sogar massive.

Nach einer Freitodbegleitung können diese in abgeschwächter Form auch vorkommen. Wenn jedoch die Angehörigen mit dem Freitod einverstanden gewesen sind (was wünschenswert, aber nicht eine Bedingung ist), ist die sog. Trauerarbeit nicht grösser als bei einem Menschen, der mit Hilfe der Medizin, der Palliativpflege oder sonst in einem guten Umfeld stirbt.

Beim Leiden und Sterben meiner Schwester - sie war 77 Jahre alt, verwitwet, kinderlos - ist nicht nur ihr engster Freundeskreis in den Entscheid einbezogen worden, sondern vor allem ich als ihr einziger Bruder, meine Frau und unsere drei erwachsenen Kinder mit ihren Ehepartnern, sowie unser bester Freund J., ein Kollege, der beruflich viel mit Sterbenden und deren Angehörigen zu tun hatte. Nur dieser Personenkreis wusste im Voraus Tag und Stunde ihres Todes. Da J. und ich von meiner Schwester in den letzten Gesprächen mit ihr gebeten wurden, sie bei ihrem Freitod zu begleiten, waren wir bei ihrem Hinschied persönlich anwesend.

Ich war dabei von meiner Familie sowie von J. und seiner Frau in hilfreichem Gedankenaustausch gut vorbereitet und verständnisvoll begleitet worden. Nach mehr als einem Jahr bin ich hundertprozentig davon überzeugt, dass der Entscheid meiner ein Leben lang gehbehinderten, nun auch noch schwer erkrankten Schwester für sie der einzig richtige gewesen ist. Sie war nicht religiös, war nicht Mitglied einer Kirche. Einen Monat vor ihrem geplanten Freitod habe ich sie gefragt, welche Gedanken sie sich über den Tod mache. Sie hat sofort geantwortet: «Ich gehe auf eine grosse Reise und ich bin neugierig, was ich erleben werde. Die Seelen sterben ja nicht.» Das hat sie ohne Emotionen, aber sehr getrost gesagt, was mich ganz fest beeindruckt und gefreut hat.

Als ihre Atmung aufhörte und bald danach ihr Herz nicht mehr schlug, sagte ich zu meinem Freund: «Wer so stirbt, der stirbt wohl.» Dies ist der letzte Satz des berühmten Liedes von Paul Gerhardt «O Haupt voll Blut und Wunden». 